\title{
Metabolomic analysis of wild and transgenic Nicotiana langsdorffii plants exposed to abiotic stresses: unraveling metabolic responses
}

\author{
Elisa Scalabrin ${ }^{1} \cdot$ Marta Radaelli $^{2}$ - Giovanni Rizzato ${ }^{1}$ - Patrizia Bogani ${ }^{3}$. \\ Marcello Buiatti $^{3}$ • Andrea Gambaro ${ }^{1,2}$ - Gabriele Capodaglio ${ }^{1,2}$
}

Received: 20 February 2015 /Revised: 30 April 2015 / Accepted: 7 May 2015 / Published online: 27 May 2015

(C) Springer-Verlag Berlin Heidelberg 2015

\begin{abstract}
Nicotiana langsdorffii plants, wild and transgenic for the Agrobacterium rhizogenes rol $\mathrm{C}$ gene and the rat glucocorticoid receptor (GR) gene, were exposed to different abiotic stresses (high temperature, water deficit, and high chromium concentrations). An untargeted metabolomic analysis was carried out in order to investigate the metabolic effects of the inserted genes in response to the applied stresses and to obtain a comprehensive profiling of metabolites induced during abiotic stresses. High-performance liquid chromatography separation (HPLC) coupled to high-resolution mass spectrometry (HRMS) enabled the identification of more than 200 metabolites, and statistical analysis highlighted the most relevant compounds for each plant treatment. The plants exposed to heat stress showed a unique set of induced secondary metabolites, some of which were known while others were not previously reported for this kind of stress; significant changes were observed especially in lipid composition. The role of trichome, as a protection against heat stress, is here suggested by the induction of both acylsugars
\end{abstract}

Published in the topical collection High-Resolution Mass Spectrometry in Food and Environmental Analysis with guest editor Aldo Laganà.

Electronic supplementary material The online version of this article (doi:10.1007/s00216-015-8770-7) contains supplementary material, which is available to authorized users.

Elisa Scalabrin

elisa.scalabrin@unive.it

1 Department of Environmental Sciences, Informatics and Statistics, University of Venice, Ca' Foscari, 30123 Venice, Italy

2 Institute for the Dynamics of Environmental Processes-CNR, University of Venice, 30123 Venice, Italy

3 Department of Evolutionary Biology, Florence University, 50127 Florence, Italy and glykoalkaloids. Water deficit and $\mathrm{Cr}(\mathrm{VI})$ stresses resulted mainly in enhanced antioxidant (HCAs, polyamine) levels and in the damage of lipids, probably as a consequence of reactive oxygen species (ROS) production. Moreover, the ability of rol $\mathrm{C}$ expression to prevent oxidative burst was confirmed. The results highlighted a clear influence of GR modification on plant stress response, especially to water deficiency - a phenomenon whose applications should be further investigated. This study provides new insights into the field of system biology and demonstrates the importance of metabolomics in the study of plant functioning.

Keywords Metabolomic $\cdot$ HPLC-HRMS $\cdot$ Nicotiana langsdorffii $\cdot$ Environmental stress $\cdot \mathrm{GR} \cdot \operatorname{rol} \mathrm{C}$

\section{Introduction}

Plants can deal with highly variable environmental stresses through the activation of a complex system of responses. When in adverse or limiting growth conditions, plants respond by activating stress-specific tolerance mechanisms at different levels of organization (transcriptomic, cellular, and physiological) in order to minimize damages while preserving enough resources for growth and reproduction. At the molecular level, abiotic stresses lead to the induction and repression of numerous genes, involving a precise regulation of the stress gene network.

Drought, high temperatures, and pollution play an important role in the current phenomenon of global climate change [1]. Heat stress conditions are known to delay the plant's growth, damage its cell membranes, denaturate proteins, increase transpiration, and reduce the opening of the stomata. One of the main observed effects of heat stress is the change in lipid composition of the cellular membranes in order to limit 
the increased membrane fluidity [2]. Water deficiency induces the inhibition of photosynthesis, the enhancement of respiration, and a lack of mineral nutrients; it also affects the lipid composition of the membrane [3]. Due to their widespread distribution and persistency, environmental contaminants, especially heavy metals, represent a relevant concern for agriculture and land use. Heavy metals such as cadmium $(\mathrm{Cd})$ and chromium $(\mathrm{Cr})$ induce enzyme inhibition, cellular oxidation, and the alteration of the metabolism [4]. $\mathrm{Cr}(\mathrm{VI})$ is the most toxic oxidation state of chromium, and its uptake is shown to influence the concentration of numerous primary and secondary metabolites [5].

Since metabolites are the end products of cellular functions, their presence and relative concentrations may be regarded as the best indicator of an organism's physiological state. Additionally, numerous secondary metabolites play a prominent role in environmental stress response as signaling molecules, protective compounds, and toxins. Antioxidants such as flavonoids, phenolics, and anthocyanins are commonly induced under both heat and chemical stresses [1], while compatible osmolytes such as amino acids, sugars, and amines [6] are produced under water, heat, and chemical stresses, balancing the plant's osmosis.

The use of genetic engineering to produce stress-resistant organisms is currently gaining in interest, especially as a means to address expected climate changes and growing food needs. Among the genetic modifications studied, the integration of the rat glucocorticoid receptor (GR receptor) showed to induce pleiotropic morphological and physiological changes in Nicotiana plants, modifying their hormonal pattern [7]; moreover, the overexpression of GR in Nicotiana tabacum leads to an increased production of different secondary metabolites and to higher resistance to nematode infections [8]. The rol genes from Agrobacterium rhizogenes have been identified as responsible for the hairy root disease in plants and induce multiple physiological and biochemical alterations in transformed plants $[9,10]$. The integration of rol $\mathrm{C}$ genes activates secondary metabolic processes and enhances the plant's response to abiotic and biotic stresses [9, 11].

The Nicotiana genus (family of Solanaceae) includes small, well-characterized plants, traditionally used as biological models for genetic and physiological studies [12]; the genetic rol C and GR modifications of $N$. Langsdorffii and Nicotiana glauca plants were previously investigated, yielding interesting results for the production of plants resistant to different stresses [7, 11, 13].

Today, the development of metabolomic methods allows scientists to simultaneously measure hundreds of compounds. Advances in analytical technologies, particularly in highresolution mass spectrometry (HRMS), along with the increased availability of new informatics tools, allowed this new discipline to rapidly grow and find a broad range of applications [14]. More than proteomics and genetics, metabolomics gives insights into the phenotypic changes of plants and the interaction between different organization levels and provides conclusive and functional information [15]. Metabolomics has found applications in a variety of fields, including the study of plant response to environmental stresses $[4,16]$. The favored method for analyzing semi-polar metabolites, which represent the main part of the plant's secondary metabolites, is high-performance liquid chromatography (HPLC) coupled to mass spectrometry (MS), which does not require derivatization prior to analysis and allows one to detect even thermolabile compounds. The Orbitrap mass analyzer, introduced in the early 2000s, has now become a mainstream mass spectrometry technique to be used in combination with an external accumulation device such as a linear ion trap.

This work presents a high-throughput metabolomic study of wild and transgenic $N$. langsdorffii plants exposed to various abiotic stresses (chromium pollution, water deficiency, and high temperature). Its aim is to identify the influence of abiotic stresses on plants and to highlight the effects of two genetic modifications (rol C and GR genes) on plant stress response. In order to achieve a comprehensive characterization of the major metabolic changes associated with genetic modifications and exposure to stress, an untargeted metabolomic analysis was performed; the use of this method enabled an extensive evaluation of plant responses and the monitoring of numerous secondary metabolites.

\section{Materials and methods}

\section{Sample preparation}

\section{Plant growth}

Specimens of $N$. langsdorffii Weinmann were cultivated in vitro in the Laboratory of Plant Genetics, Department of Evolutionary Biology of the University of Florence. Plant genotypes were multiplied by withdrawing portions of stems containing the internodes. Plants were grown in a specific growth medium LS (MSM0-Sigma Aldrich ${ }^{\circledR}$, Buchs, Switzerland) containing sucrose and plant agar (LAB Associated, BV, The Netherlands) in proportion of 3 and $0.6 \%$ respectively; $\mathrm{pH}$ conditions were comprised between 5.6 and 5.8. Plants were grown for 30 days in a growth chamber at a temperature of $24 \pm 1{ }^{\circ} \mathrm{C}$ with a photoperiod of $16 \mathrm{~h}$ and at a relative humidity of $80 \%$. Plants were then fastcleaned with distilled water to remove the growth medium residues and frozen in liquid nitrogen; samples were then freeze-dried in an Edward freeze-drying machine and, after complete water evaporation, maintained at room temperature. Samples were grinded and homogenized by using a ball mill (MM 400, Retsch, Verder Scientific, Haan, Germany) 
equipped with two PTFE vessel and grinder balls; samples were ground for $5 \mathrm{~min}$ with a vibration frequency of $20 \mathrm{~Hz}$ to achieve a final fineness of $\approx 5 \mu \mathrm{m}$.

\section{Genetic modifications}

Wild-type (WT) plants were genetically modified by insertion of the gene codifying for the rat GR receptor and the rol $\mathrm{C}$ gene from $A$. rhizogenes. The procedure for obtaining these genetic modifications is extensively described by Giannarelli et al. [7] and Fuoco et al. [13]. The expression of the transgenes was screened, as previously described [13].

\section{Stress inductions}

The plants were exposed to three different abiotic stresses: heat stress (HS), water stress (WS), and chemical stress by chromium (CR). HS was induced on WT, Rol C, and GR plants by exposing them to a temperature of $50{ }^{\circ} \mathrm{C}$ for $2 \mathrm{~h}$ into a thermostatic chamber (SANYO mod.MIR-153, Panasonic, Moriguchi, Osaka, Japan). After the treatment, the plants were collected and freeze-dried. Some preliminary studies were conducted on $N$. langsdorffii WT plants in order to identify the best temperature for the heat treatment. Temperatures of $35,44,46,48$, and $50{ }^{\circ} \mathrm{C}$ were tested for different periods of time, and the final parameters were selected on the basis of the ionic release and recovery ability, which is the percentage of survival of treated plants after their incubation at $24{ }^{\circ} \mathrm{C} \pm 1{ }^{\circ} \mathrm{C}$ for 20 days. WS was simulated by adding polyethylene glycol 6000 (PEG 6000) into the growth medium, thereby reducing the osmotic potential of the LS medium. Different concentrations of PEG have been tested, and the addition of $20 \%$ was selected on the basis of the growth ability of WT plants. Exposure to heavy metal stress was performed, as described by Del Bubba et al. [11], and the $\mathrm{Cr}(\mathrm{VI})$ concentration was determined on the basis of preliminary experiments on stress response, as previously described [13].

\section{Metabolomic analysis}

\section{Sample treatment procedure}

The sample treatment procedure was based on the protocol of De Vos et al. [15]. Twelve different typologies of samples were analyzed: non-stressed wild and transgenic plants (WT, RolC, and GR), HS plants (WTHS, RolCHS, and GRHS), WS plants (WTWS, RolCWS, and GRWS), and CR plants (WTCR, RolC-CR, and GRCR). The samples were produced as mixed plant material deriving from different organisms of the same typology in order to reduce the biological variability associated with the single plants. Each sample was analyzed in three replicates to check the repeatability of the experiment. Blank samples and mixed samples, consisting of a pool mixed material of all the typologies of samples analyzed, were prepared and used to control contamination and repeatability. An internal standard (IS), phenyl- ${ }^{13} \mathrm{C}_{6}$ Salicylic acid, was used in order to check for the repeatability of the sample treatment procedure. The lyophilized and milled plant material $(50 \pm$ $0.5 \mathrm{mg}$ ) was weighted, and the IS was added at a concentration of $19.4 \mu \mathrm{g} / \mathrm{g}$. After homogenization, the samples were treated for $30 \mathrm{~min}$ in an ultrasonic bath with $1.5 \mathrm{~mL}$ of $\mathrm{MeOH} / \mathrm{H}_{2} \mathrm{O}$ 75:25 $(v / v)$ acidified with formic acid $0.1 \%$ and then centrifuged for $20 \mathrm{~min}$ at 14,000 rpm; the supernatant was collected and filtered with PTFE syringe filters (Ø $25 \mathrm{~mm}, 0.2 \mu \mathrm{m}$ ). Before use, the filters were activated with $2 \mathrm{~mL}$ of $\mathrm{MeOH}$ and $1 \mathrm{~mL}$ of ultrapure water and dried under a $\mathrm{N}_{2}$ flow. The solution obtained was finally analyzed.

\section{Instrumental method}

Analyses were performed by an UltiMate 3000 (Dionex) coupled to an ESI-LTQ Orbitrap XL (Thermo Fisher Scientific, Waltham, USA). The chromatographic column used was a SB-Aq Narrow Bore RR 2.1×150 mm, $3.5 \mu \mathrm{m}$ (Agilent Technologies, Wilmington, USA); the gradient elution was carried out with a mobile phase constituted by $\mathrm{H}_{2} \mathrm{O}$ acidified with $0.01 \%$ of formic acid (eluent A) and ACN acidified with $0.01 \%$ of formic acid (eluent B). The chromatographic run included an initial 5-min isocratic phase at $100 \%$ of eluent A, followed by a 40-min gradient until $100 \%$ of eluent B composition; it then continued with 15-min isocratic in organic phase and a final 15-min step of column conditioning at the initial eluent proportions. The eluent flow was $200 \mu \mathrm{L} / \mathrm{min}$, and the sample injection volume was $5 \mu \mathrm{L}$. To check instrumental reproducibility, the mixed samples were analyzed throughout the sample series, verifying the intensity of the signal. The ESI source was operated in both negative and positive polarities; the capillary temperature was set at $275^{\circ} \mathrm{C}$ and the vaporization temperature at $300{ }^{\circ} \mathrm{C}$; and the sheath, auxiliary, and sweep gas were flown at 35, 5, and $0 \mu \mathrm{L} / \mathrm{min}$, respectively. The analyses were conducted in fullscan modality at a resolving power of 60,000 , with a mass range between 90 and $1500 \mathrm{~m} / \mathrm{z}$. Data-dependent acquisitions were also performed in order to obtain a complete fragmentation pattern of the molecules. Measurements were carried out by means of the internal calibration method (reference mass) to correct automatically the mass calibration for each scan, obtaining a mass accuracy of $2 \mathrm{ppm}$. The intensity of the reference mass was monitored over samples to assure constant and reproducible ionization.

\section{Data processing and metabolite identification}

The mass spectra alignment and baseline correction were carried out by MetAlign [17, 18], and the mass signal clustering was conducted by MSClust [19] to obtain reconstructed 
metabolites for further data analyses. The best processing parameters were selected on the basis of the spectra characteristics and through several experimental tests in which the presence of peak splitting, the incorrect removal of relevant peaks, the level of noise, and the eventual misalignment were checked. The list of masses produced as output by MetAlign was partially processed by MS Excel in order to properly prepare it for MSClust processing. Multivariate analysis, particularly principal component analysis (PCA), was finally performed by means of Statistica 8.0 (StatSoft, Inc., 2007); hierarchical cluster analysis (HCA) was carried out using the heatmap.2 function as implemented in the R 2.14.2 software version (www.R-project.org). The metabolite intensities before statistical analysis were normalized using $\log _{2}$ transformation and standardized using range scaling. Metabolite identification was performed by means of available online libraries (Metlin, HMDB, Dictionary of Natural Products, and LIPID MAPS Structure Database) and literature, considering the monoisotopic mass, the most probable molecular formula, and the fragmentation pattern. The identification level was assigned according to [20].

\section{Results and discussion}

\section{Metabolomic profile of $N$. langsdorffii plants}

The metabolomic analysis of $N$. langsdorffii samples aimed to obtain a complete profiling of the metabolites affected by stress exposure. The extraction reproducibility was checked by means of the IS, which intensity resulted comparable in all samples (CV\% 7.9). The technical reproducibility was assured by the use of internal calibration method and the monitoring of mix sample intensities all throughout the sample sequence (see Electronic Supplementary Material (ESM), Figures S1, and S2). The chromatograms obtained from the HPLCHRMS analysis were processed by means of MetAlign for unbiased mass peak picking and baseline correction; the resulting peaks were further handled with MSClust, grouping the mass signals into more than 700 reconstructed metabolites. A final number of 204 compounds were putatively annotated; the complete list of which is reported in the supporting information (ESM Tables S1, and S2). Negative and positive representative chromatograms, with the main classes of identified metabolites, are reported in Fig. 1a, b. PCA was then carried out on all the detected compounds (Fig. 2), highlighting the separation of plants mainly on the basis of the applied abiotic stress. Samples subjected to HS appeared well separated from the others, suggesting a significant metabolic change associated with this kind of stress. Non-stressed plants (controls) also clustered well, being separated from HS samples by the first component. CR samples showed higher internal variability and showed low variable coefficients in both factors.
Remarkably, GRWS samples presented high distance in both first and second factors (Fig. 2) from RolCWS and WTWS samples, indicating clear differences in their metabolomic profile. GRWS samples seemed to be more similar to nonstressed samples, suggesting an influence of the GR genetic modification on the plant's response to water deficit. PCA and the heat map (Fig. 3) highlighted the presence of clear groups of metabolites with high intensities in HS, CR, and WS samples. This analysis enabled the selection $(t$ test with a $p$ value $<$ 0.001 ) and identification of the main key compounds of the different samples.

\section{Heat stress}

The principal classes of compounds which showed relevant changes in heat stress (HS) plants were lipids, acylsugars, and glykoalkaloids.

The lipid composition of membranes is known to be affected, especially in the leaves, after long-term heat exposition (e.g., 1 month, $35{ }^{\circ} \mathrm{C}$ ) [2]. Although our heat experiment consisted of a short-term exposure $(2 \mathrm{~h})$ at very high temperature $\left(50{ }^{\circ} \mathrm{C}\right)$, the relatively short time during which plants sustained the stress conditions affected some plant metabolites. Among the lipid classes, the compounds which showed the most significant changes were sulfoquinovosyl monoacylglycerols (SQMG), sulfoquinovosyl diacylglycerols (SQDG), mono- and di- galactosyldiacylglycerols (MGDG and DGDG), and phosphatidylglycerol (PG) (see Fig. 4a-f and Tables S3 and S4 in the ESM); these compounds represent the main constituents of thylakoid membranes [21]. Such changes in lipid composition can be seen as the first response of the plant system to high temperature stress. The levels of sulfolipids and galactolipids increased especially in WTHS and RolCHS plants. The ratio of lipids in HS samples, compared to the controls, varied from a minimum of 1 to a maximum of 54 in GR plants, while in RolC they ranged from 1 to 245, and in WT from 1 to 103 . As previously outlined, the GR genetic modification seems to influence the plant's metabolism, conferring it a higher resistance against various kinds of stresses. In this case, too, GR plants presented a different behavior from both WT and RolC; the increases of lipids in GRHS samples were statistically different from those in WTHS plants for 15 compounds, in comparison to 7 statistically significant changes between RolCHS and WTHS. The lower increases in lipids observed in GR plants could indicate a lower damage of the photosynthetic system and chloroplast structure; however, more studies are necessary to confirm this hypothesis. The stabilization function of the photosynthetic system, carried out by sulfolipids and galactolipids, was suggested by several studies [21, 22]. Sulfolipids are known to accumulate during high/low temperature stress and water deficit conditions [23]. Our results are in agreement with those reported by Chen et al. [22] about Arabidopsis plants. Another 
Fig. 1 Negative (a) and positive (b) representative total ion current (TIC) plots with the main classes of identified metabolites

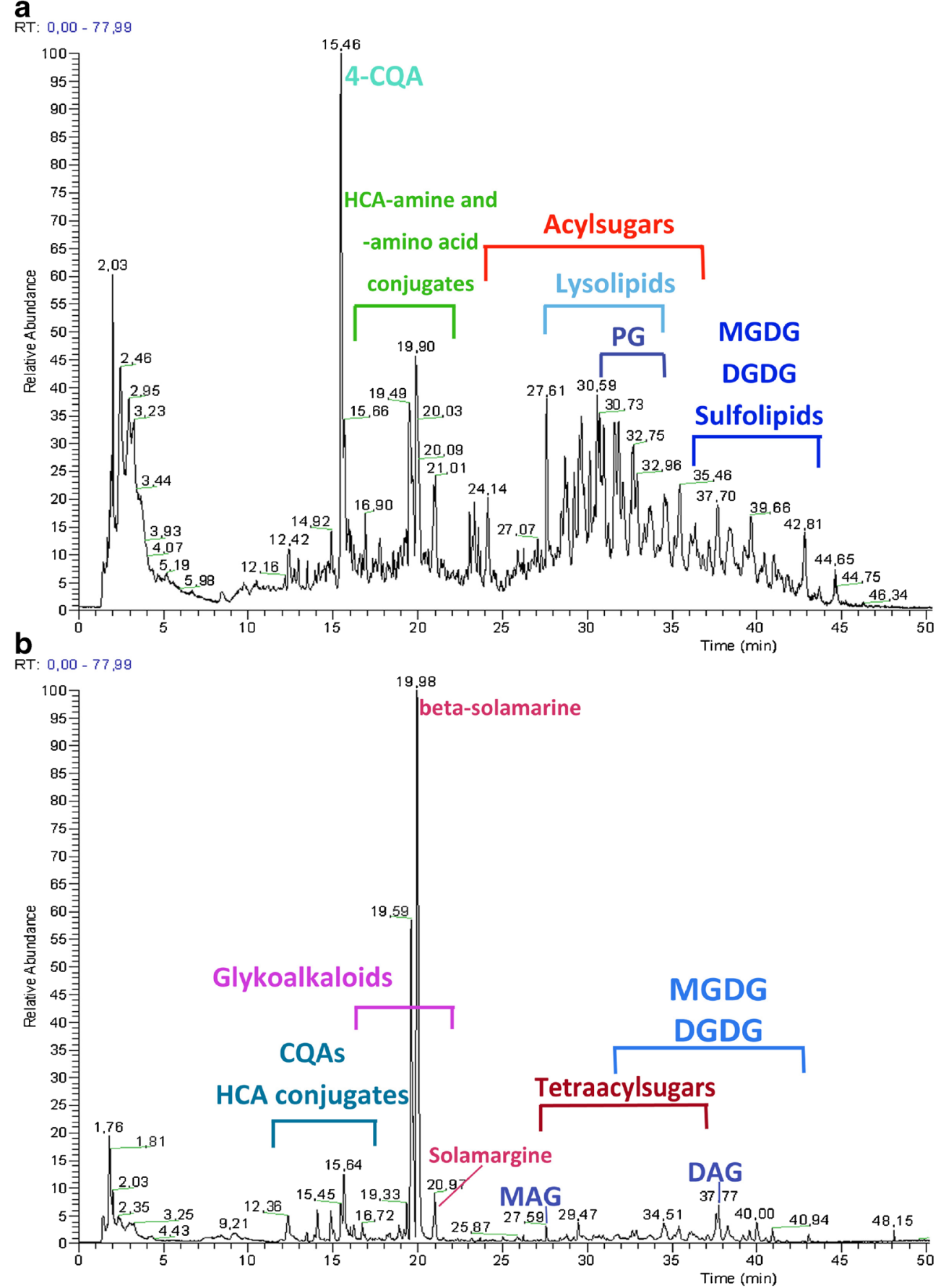

lipid that was found to increase in HS samples was phospatidylethanolamine (PE), which is one of the main constituents of the mitochondrial membrane, where respiration takes place. Previous studies showed that, in the first phase of HS, the respiration rate rapidly increases with temperature while drastically decreasing after an extreme tolerable temperature [6]. The observed increase in PE (Fig. 4d-f and Tables S3 and S4 in the ESM) could therefore be associated to the initial enhanced respiration activity, as previously suggested by Otsuru et al. [24].
Three acylglycerols, which are minor cell components widely distributed in all plant tissues, showed increases of 20-60-folds in HS samples. However, unlike other lipids, they presented lower increases in RolC plants. These components are generally not very abundant in cells because of their surfactant properties, which can determine the disruption of the cell membrane. Mono- and di-acylglycerols are the precursors of MGDG/DGDG and SQDG in chloroplasts and of PE in the endoplasmatic reticulum. Their presence and function have not been related yet to any plant response to stress. 
Fig. 2 Score plot of principal component analysis of all the metabolites detected in Nicotiana langsdorffii samples

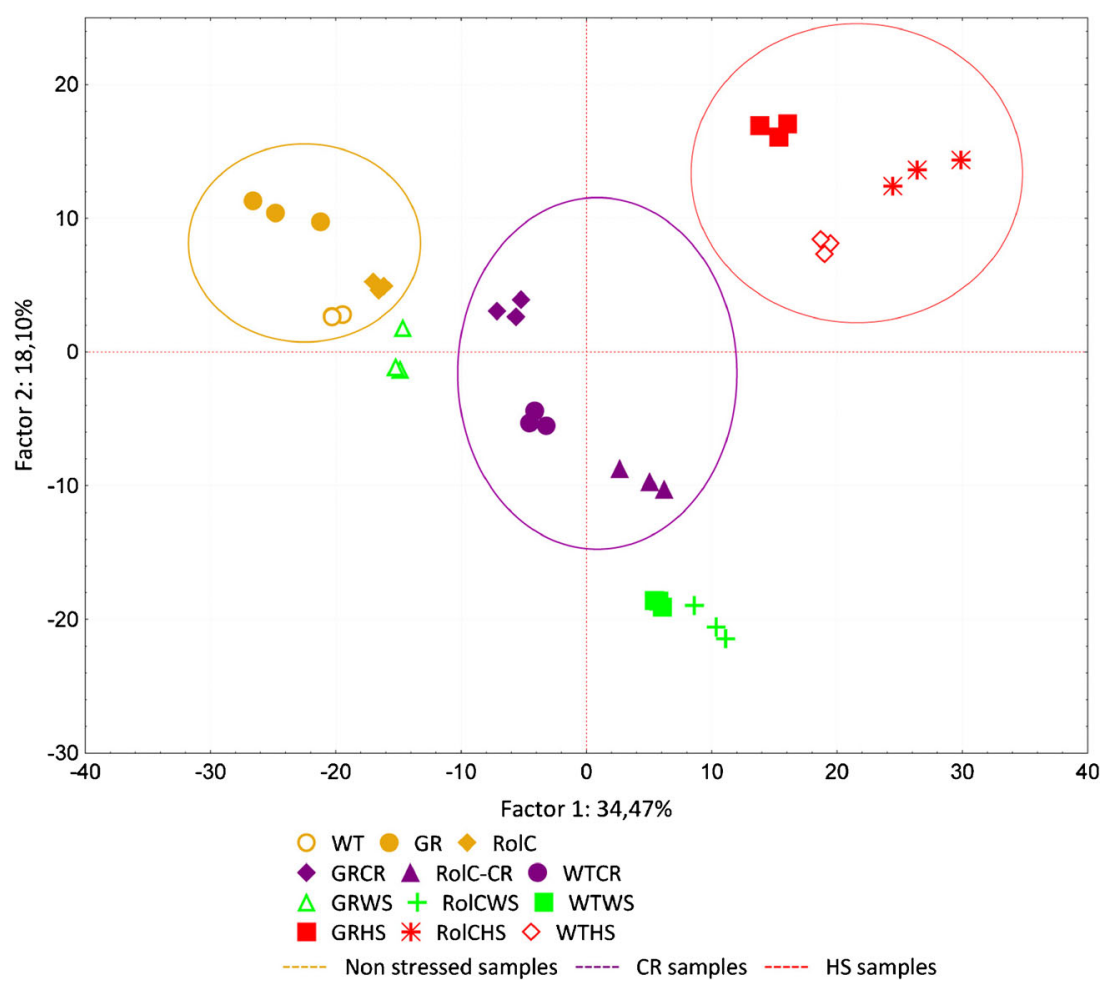

Acylsugars are non-volatile metabolites that constitute a significant proportion of leaf biomass in some Solanaceous species, such as Nicotiana. They are known to be involved in the defense against pathogens and herbivores [25]. Acylsugars are mainly produced and accumulated in the plant's trichomes, thin structures outgrowing from leaves, and stems in which many specialized secondary metabolites and proteins are produced and stored, especially with defense functions [26, 27]. The increase in trichome density has already been positively correlated to temperature increase and water decrease, probably because trichomes favor the reflection of sunlight radiation, as well as heat dissipation and water transpiration [28]; however, to our knowledge, no study so far has investigated the possible relation between acylsugars and HS response. Our results suggest that these compounds, besides their role against biotic stress, could also take part in the response to abiotic stresses, especially heating or drought. Twenty acylsucroses were detected, 17 of which showed significant increases in HS samples with respect to the controls. Most of these compounds were tetra-acylsucroses (11 compounds), followed by 7 tri-acylsucroses and two penta-acylsucroses. The higher increases under HS were observed for tetraacylsucrose S4:20 (where 4 is the number of acyl groups and 20 the number of carbons) and penta-acylsucrose S5:25. The acylsugar ratios between HS samples and the controls were 20,35, and 16 for penta-acylsucrose (S5:25) and 19, 6, 12 for tetra-acylsucrose (S4:20) in GRHS, RolCHS, and WTHS, respectively. The other compounds showed similar increases in all the HS samples, except for tetra-acylsucrose
(S4:17), tetra-acylsucrose (S4:18), and tetra-acylsucrose (S4:19), which presented higher increases in GRHS samples.

Another relevant class of compounds identified in HS samples was the steroidal glycoalkaloid group derived from solasodine and tomatinedol; these compounds and their glycosides are known constituents of Solanaceae plants and studied for their toxicity in potatoes [29] and for their insecticidal potential [30]. Eleven solamarines, solamargines, and solasodines, or their derivatives, were identified, and their intensities proved comparable in WT, GR, and RolC plants. The difference in glykoalkaloid content between control and HS samples was significant at a $p$ value $<0.05$. $\delta$-Solamarine and $\delta$-solamarine deoxyhexose showed the highest increases in WTHS and RolCHS samples, with a ratio of 8 and 6 , respectively, while solamargine presented the highest increase in GRHS samples, being 9 times higher than in controls. The increases in the concentrations of glykoalkaloids in plants exposed to HS agreed with the study by Coria et al. [31], which showed that high $\alpha$-solanidine content in potato tubers could be related to exposure to high temperature (immersion in hot water at $35{ }^{\circ} \mathrm{C}$ for $4 \mathrm{~h}$ ). Other studies, moreover, reported enhanced total glykoalkaloid levels in potato tubers after exposure to heat/drought $[32,33]$.

\section{Chromium and water stresses}

Amino acid derivates, hydroxycinnamic acids (HCAs), and their polyamine conjugates appeared to be the most relevant compound classes in plant response to chemical stress 
Fig. 3 Heat map of the metabolites detected in Nicotiana langsdorffii samples. A colorcoded matrix represents the intensities of the reconstructed metabolites in the three replicates of each plant typology. Prior to statistical analysis, the values have been $\log _{2}$ transformed and normalized
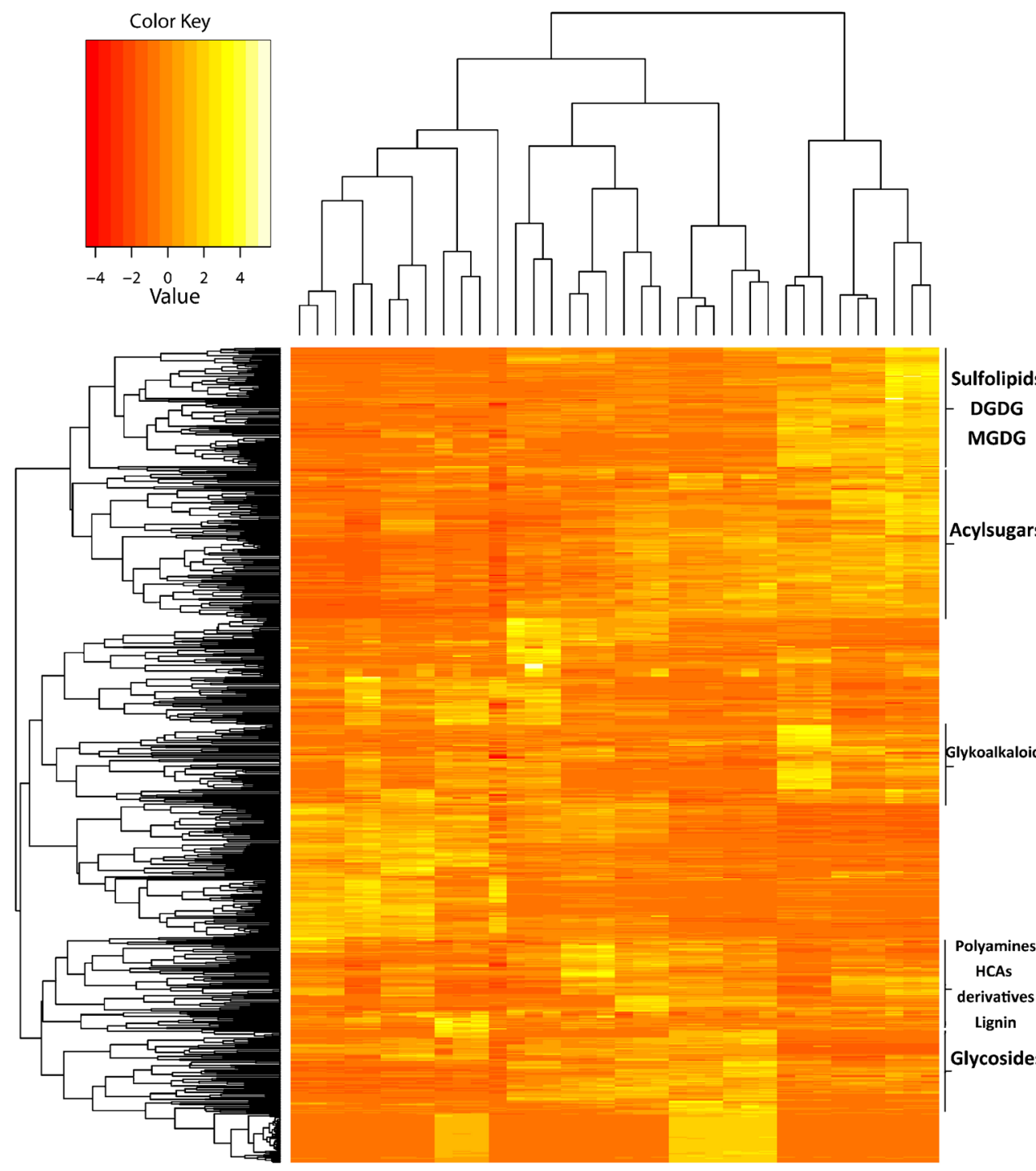

MGDG

Acylsugars

A
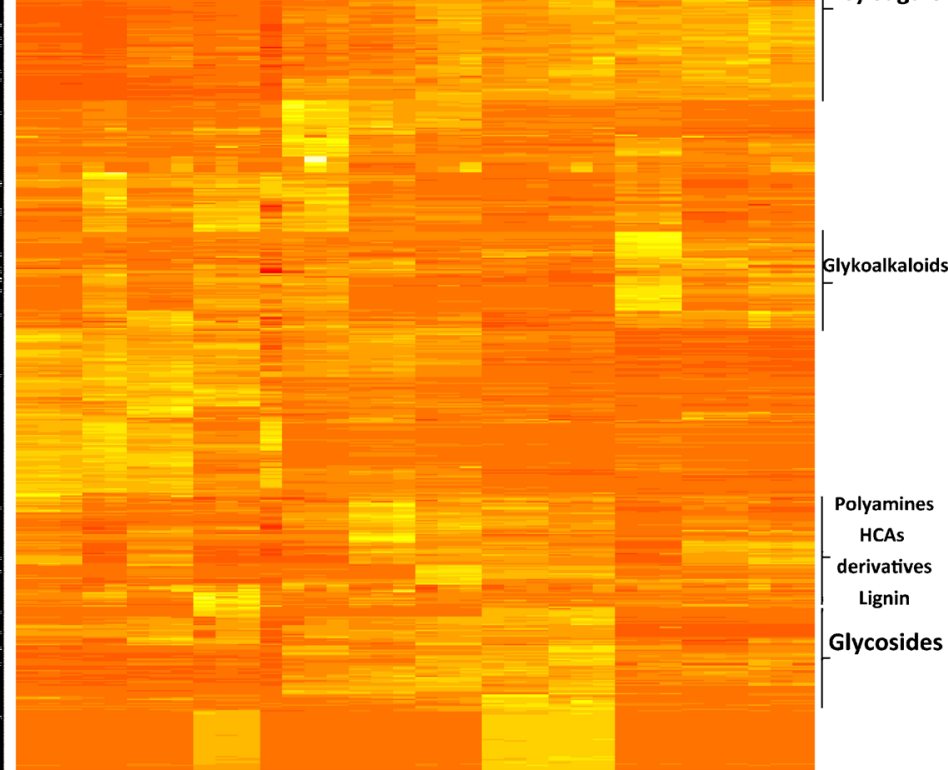

Polyamines

HCAs

derivatives

Lignin

Glycosides
(Fig. 5a-c and Tables $\mathrm{S} 3$ and $\mathrm{S} 4$ in the ESM), as suggested in previous studies [4]. The 4-caffeoylquinic acid (4-CQA) showed high induction in all CR stressed samples, while other isomers did not present a similar behavior. Del Bubba et al. [11] reported similar changes in the shoots of $N$. langsdorffii exposed to $\mathrm{Cr}(\mathrm{VI})$. However, they revealed increased levels also for 3- and 5-caffeoylquinic acids (3-CQA and 5-CQA). In addition to 4-CQA, several HCA-amino acid and HCAamine conjugates increased in $\mathrm{CR}$ samples, such as feruloylaspartic acid, dicaffeoyl spermidine, glucopyranosyl-caffeoyl putrescine, and bis-dihydrocaffeoyl spermine. Amino acids, especially proline, alanine, and serine, are known to accumulate in plants exposed to salinity stress as compatible solutes in order to maintain the osmotic equilibrium of cells. A similar stabilizing role is also played by polyamines (spermidine, putrescine, and spermine); HCA-amine conjugates, moreover, have a high antioxidant potential and are known to be part of the plant defense system against ROS molecules and abiotic stresses [1]. Biosynthesis of polyamines is known to be induced during WS [34]. It is worth noting that the metabolic pattern of GRCR samples was less affected by stress in comparison to other stressed samples; GRCR showed increased levels of conjugated amines. All the CR-stressed plants showed enhanced levels of grossamide, a lignin amide, which could indicate an induction of the lignin biosynthetic pathway as a consequence of chemical stress. The latter observation agrees with the study of Van de Mortel et al., who suggested that lignin biosynthesis prevents excess efflux of metals from the vascular system [35]. Besides these compounds, lipids and acylsucroses showed relevant changes in plants exposed to chemical stress by chromium, in comparison to the controls. Tetra-acylsucroses (S4:19, S4:24, and S4:26) presented 


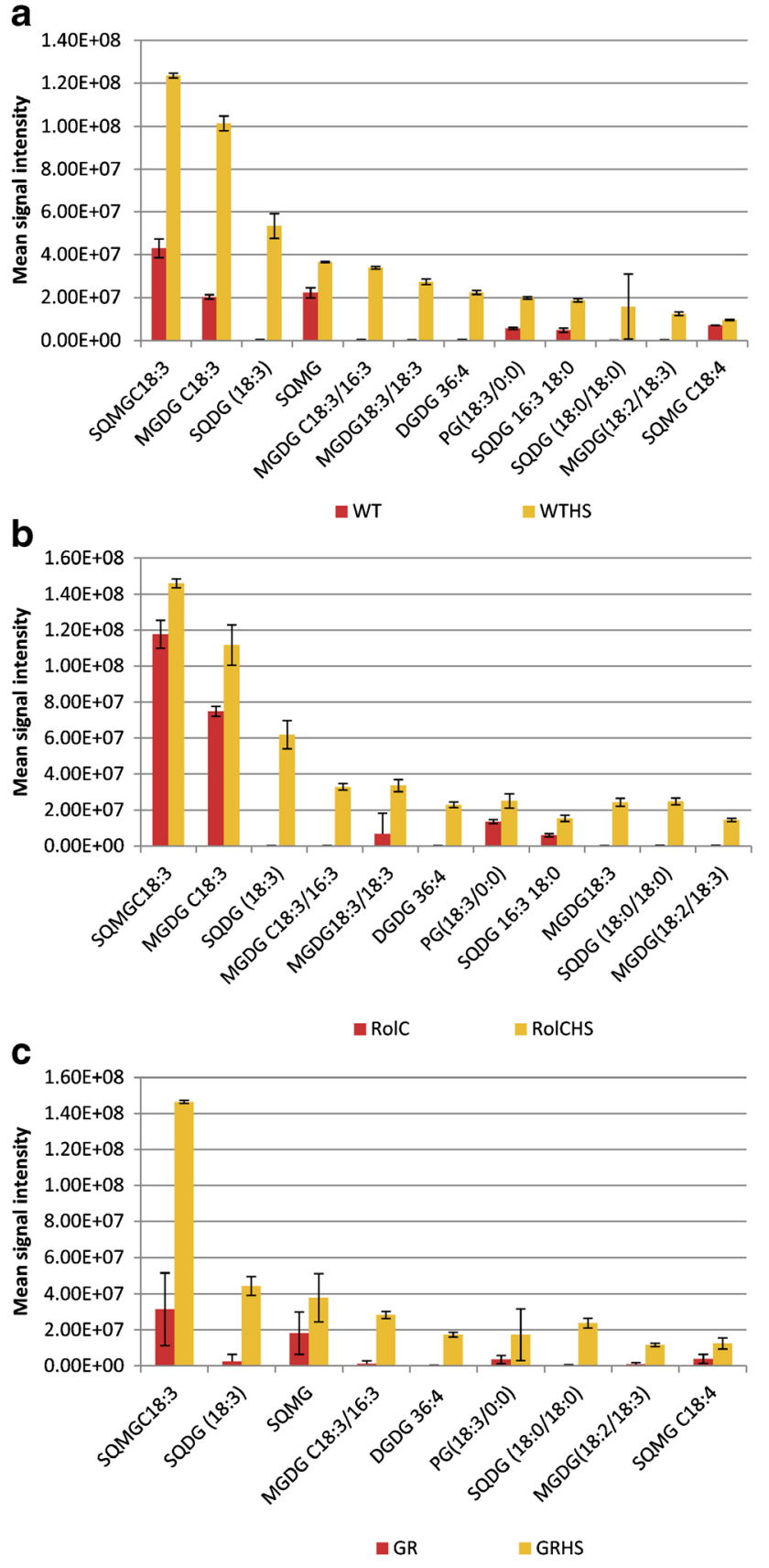

Fig. 4 Levels of the principal SQMG, SQDG, MGDG, and DGDG, identified in negative polarity, showing the highest increases in WTHS (a), RolC-HS (b), and GRHS (c) samples in comparison to the controls. Levels of MGDG, DGDG, PG, and PE, identified in positive polarity,

significant increases in all WTCR, GRCR, and RolC-CR samples, with intensities ranging from 9- to 136-fold the controls. WTCR samples showed the highest number of metabolites with significant changes (Fig. 5a); among these, a high number of structurally different lipids such as sphingolipids, fatty acids, phospholipids, SQMG, and lysolipids were observed (Tables S3 and S4 in the ESM). Hydroxylated fatty acids, namely trihydroxyoctadecenoic acid and hydroxylinoleic

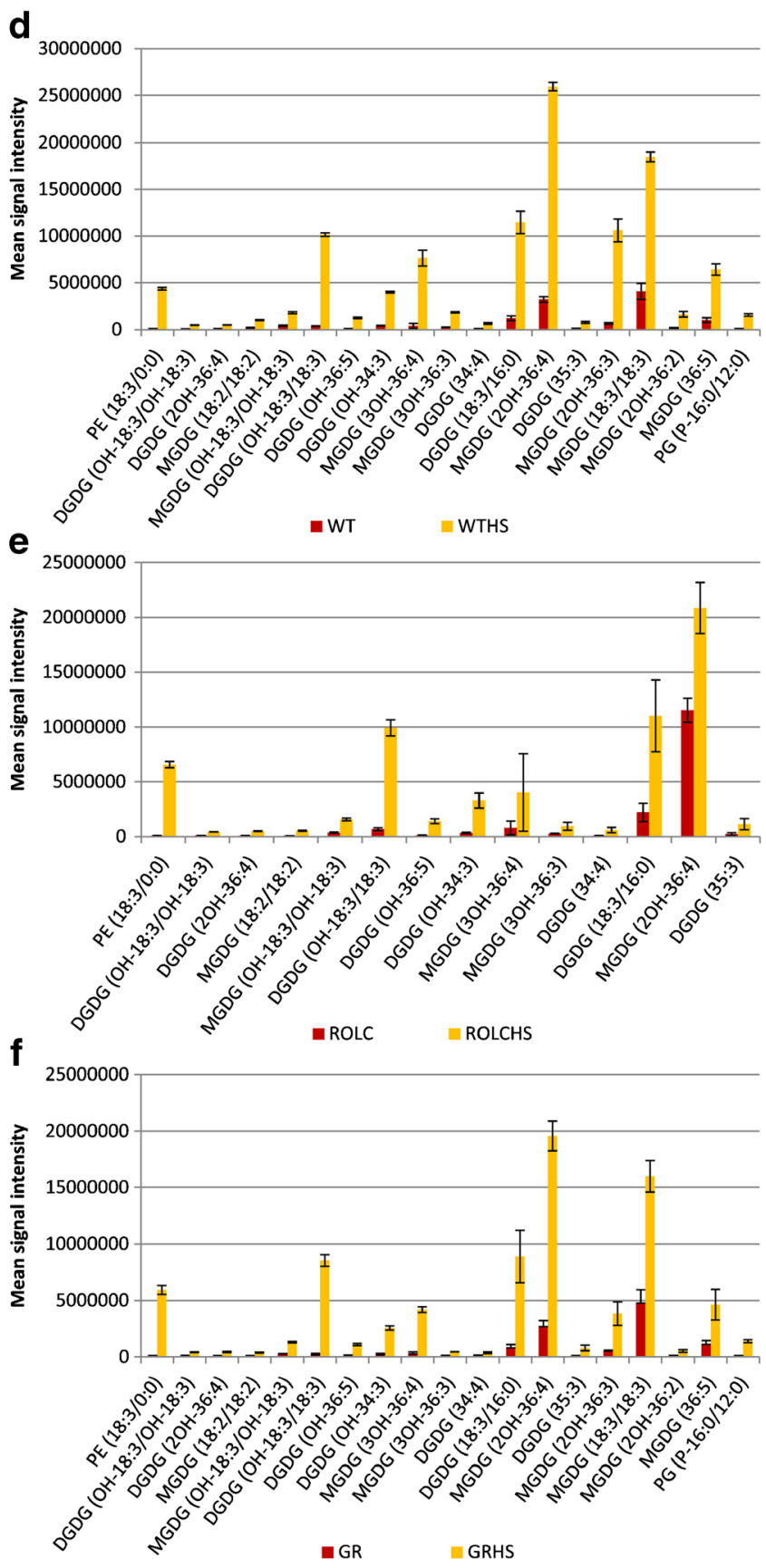

showing the highest increases in WTHS (d), RolC-HS (e), and GRHS (f) samples compared to the controls. Error bars represent the standard deviations

acid, were observed; the increases of these compounds are probably related to the production of ROS molecules as a consequence of heavy metal stress. ROS compounds are involved in stress response, and they generally determine the peroxidation of lipids, generating oxylipins such as, for instance, phytohormone jasmonic acid [36]. Most of the lipids that showed significant increases in CR plants were different from those discussed above for HS experiments. Interestingly, 
Fig. 5 Compounds showing statistically significant changes in WTCR (a), RolC-CR (b), and GRCR plants (c) identified in negative polarity. Error bars represent the standard deviations
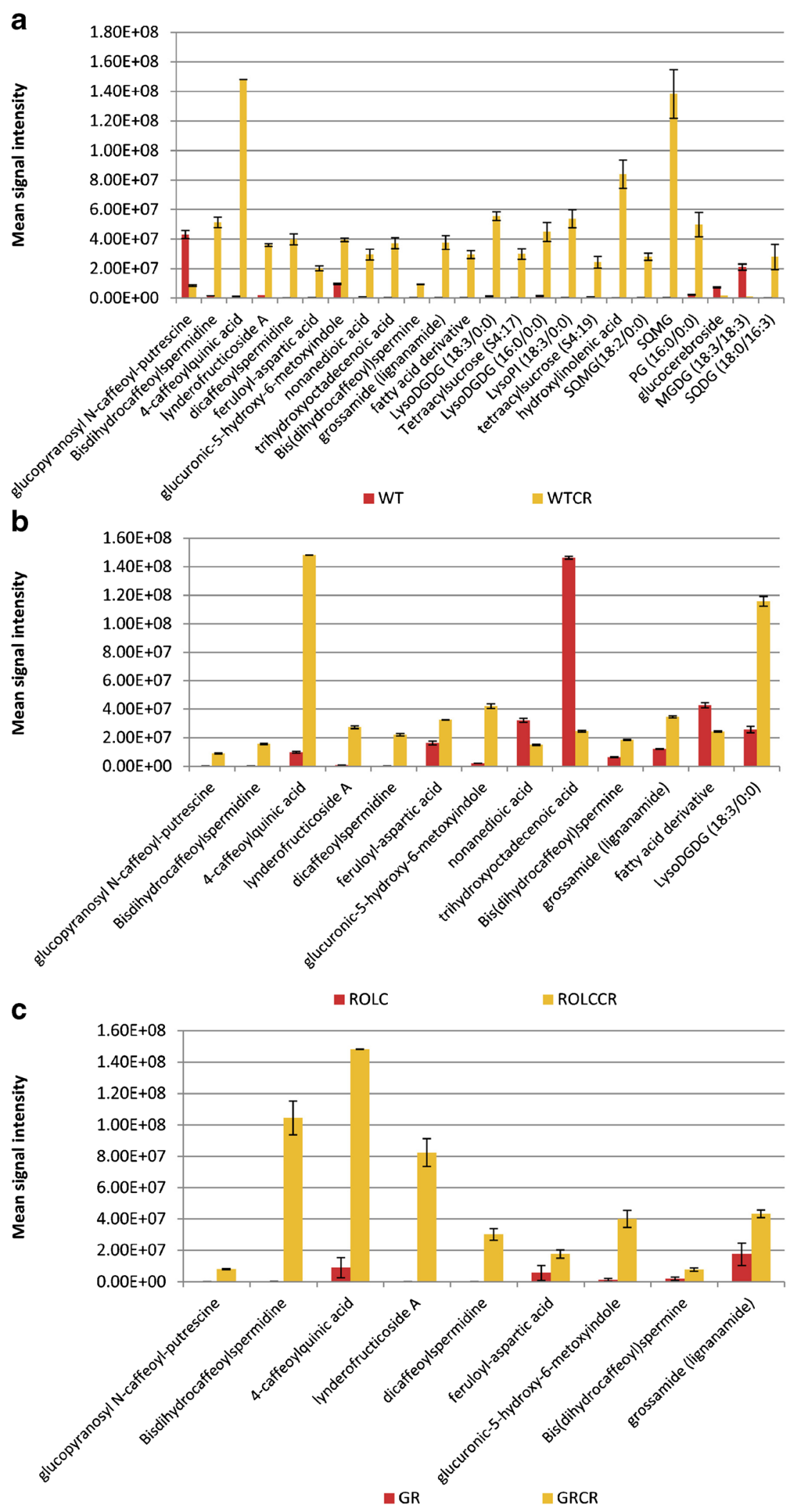

indeed, increases in some lysolipids, namely lysoPE, lysoDGDG, and lysophosphatidylinositol (lysoPI), were observed. The production of lysophospholipids was previously associated to herbivore stress and wounding. This effect was explained as an induction of the release of polyunsaturated fatty acids to be used for oxylipin production [16]; this result 
is consistent with the fatty acid hydroxylation in WTCR samples discussed above [37]. The response of RolC plants was opposite in this case, showing decreased levels of fatty acids and hydroxyl fatty acids in CR samples compared to controls. Indeed, this result confirmed that the rol $\mathrm{C}$ gene expression prevents the production of ROS, as previously reported by Bulgakov et al. [38] for Rubia cordifolia cells. GRCR plants showed no significant changes in lysolipid intensities or in fatty acid content. These findings strongly indicate an effect of RolC and GR genetic modifications on the plant's response to chemical stress; however, the effect of the two genetic modifications appears significantly different.

Although the complete metabolite profiling in positive polarity of water-stressed samples was not possible, due to the interferences caused by the PEG 6000 , the negative polarity analysis yielded several interesting findings (Table 1). WT samples presented the highest ratios of metabolites, for different classes of compounds, between stressed and non-stressed samples. Lipids, hydroxyl and peroxyl fatty acids, lignins and lignanamides (syringaresinol and grossamide), terpenes (capsianoside II and prieuriarin), malonic acid derivatives, indolizidine alkaloids (ipalbidine), carbohydrate conjugates (primeveroside) and polyamines were the main metabolites affected. This result could suggest that the response to WS involves not only a few specific metabolic pathways, as in the case of chemical stress and HS, but a greater number of processes. The effect of the hydroxylation and peroxidation of lipids was similar to the one previously observed for $\mathrm{Cr}(\mathrm{VI})$ stressed samples and is probably related to the presence of ROS [34]. The highest increases in WTWS samples were registered for hydroxyperoxyoctadecatrienoic acid, trihydroxyoctadecenoic acid, glucopyranosyl-penta malonic acid and bis-hydrocaffeoyl spermine. RolCWS samples were mainly characterized by increased levels of antioxidant such as lignins, HCA derivatives and terpenes. In the case of WS, too, GR response seemed to be limited in comparison to WT and ROLC plants, showing statistically significant increases only in a few compounds, with ratios ranging from 2 to 56 . Interestingly, WS induced the production of 3-CQA in all GRWS, WTWS and RolC-WS samples, but resulted in no increased amounts of 4-CQA.
Table 1 Mean ratios and standard deviations $\left(\sigma^{2}\right)$ between WS samples and controls. Values are reported only for statistical significant changes ( $p$ value $<0.05$ ) while not relevant changes are indicated with n.s

\begin{tabular}{|c|c|c|c|c|c|c|}
\hline Metabolites & $\begin{array}{l}\text { Mean } \\
\text { GRWS/GR }\end{array}$ & $\sigma^{2}$ & $\begin{array}{l}\text { Mean } \\
\text { RolCWS/RolC }\end{array}$ & $\sigma^{2}$ & $\begin{array}{l}\text { Mean } \\
\text { WTWS/WT }\end{array}$ & $\sigma^{2}$ \\
\hline Trihydroxyacetophenone & n.s. & n.s & 96 & 15 & n.s & n.s \\
\hline 3-Caffeoylquinic acid & 5 & 2 & 134 & 4 & 5 & 0.1 \\
\hline Glucopyranosylpentylmalonic acid & n.s & n.s & 27 & 1 & 500 & 3 \\
\hline Benzyl-primeveroside & n.s & n.s & 138 & 40 & 166 & 9 \\
\hline lysoPE (18:4/0:0) & 47 & 18 & 8 & 2 & 2 & 0.01 \\
\hline Feruloyl aspartic acid & n.s & n.s & 2 & 0.03 & 33 & 1 \\
\hline Feruloyl tyramine & n.s & n.s & 115 & 9 & 5 & 0.1 \\
\hline Glucosylated syringaresinol & 6 & 3 & 90 & 219 & 256 & 36 \\
\hline$\gamma$ Solamarine +2 deoxyhexoses & n.s & n.s & 42 & 0.5 & 2 & 0.1 \\
\hline Prieurianin & n.s & n.s & 108 & 4 & 4 & 0.05 \\
\hline Capsianoside II & 5 & 1 & 79 & 2 & 88 & 0.1 \\
\hline Grossamide (lignanamide) & n.s & n.s & 78 & 2 & 44 & 0.05 \\
\hline Trihydroxyoctadecenoic acid & n.s & n.s & n.s & n.s & 425 & 3 \\
\hline Glucopyranosyl ipalbidine & n.s & n.s & 16 & 0.2 & 147 & 20 \\
\hline bis(dihydrocaffeoyl)spermine & 2 & 2 & 18 & 1 & 378 & 65 \\
\hline Hydroxyperoxyoctadecatrienoic acid & n.s & n.s & 23 & 0.5 & 284 & 6 \\
\hline Fatty acid derivative & n.s & n.s & 0.3 & 0.01 & 93 & 7 \\
\hline lysoPE (18:3/0:0) & n.s & n.s & 21 & 194 & 173 & 30 \\
\hline Tetraacylsucrose (S4:17) & n.s & n.s & n.s & n.s & 66 & 39 \\
\hline Hydroxylinolenic acid & n.s & n.s & n.s & n.s & 91 & 4 \\
\hline Quinquenoside I & n.s & n.s & 18 & 225 & 64 & 0.1 \\
\hline Triacylsucrose (S3:22) & n.s & n.s & 61 & 288 & 110 & 6 \\
\hline Glucocerebroside & n.s & n.s & 16 & 339 & 11 & 1 \\
\hline Hydroxy pheophorbide A & n.s & n.s & 8 & 1 & 46 & 0.2 \\
\hline MGDG (18:3/16:2) & n.s & n.s & 12 & 4 & 91 & 6 \\
\hline SQDG (18:0/16:3) & n.s & n.s & n.s & n.s & 102 & 12 \\
\hline
\end{tabular}




\section{Conclusions}

This work presented an untargeted metabolomic study of the stress responses in wild and transgenic $N$. Langsdorffii plants. The metabolomic analysis enabled a complete evaluation of the plant's metabolic status under abiotic stress conditions. More than 200 metabolites, most of which belonging to the class of lipids such as fatty acids, sulfolipids, PG, PE, acylglycerols and galactolipids, were identified. The presence of many acylsugars and steroidal glycoalkaloids (solasodine and tomatinedol derivatives) was also detected, indicating significant changes between stressed and control plants.

The extended characterization of the metabolic pattern highlighted several interesting differences among the genotypes.

Wild plants showed the highest metabolic changes after stress exposure, demonstrating to be more affected than transgenic plants. HS exposure strongly influenced the metabolic profile of plants. All the HS samples showed increases in lipidic compounds, especially MGDG, DGDG and sulphoquinovosyl derivatives; these compounds are known to protect the photosynthetic system and to be enhanced in conditions of increased temperatures [21,23]. An accumulation of acylsucroses, trichome-specific Nicotiana compounds, and glykoalkaloids was also observed after HS and WS exposure; on the basis of these results, we can hypothesize that trichomes, which are known to be involved in pathogen defense, could play a role also in abiotic stress response [28]. All plants exposed to $\mathrm{Cr}(\mathrm{VI})$ stress showed an increase in levels of antioxidant compounds, such as HCA derivatives and lignins, suggesting an induction of the shikimic acid pathway. The effects of chromium stress were stronger in RolC and WT plants, which showed the presence of hydroxyl- and peroxylipids, while effects were reduced in GRCR plants, where only the 4-CQA and HCA-amine conjugate levels were affected. RolC-CR and WTCR plants also showed the presence of lysolipids, as a result of the induction of the release of polyunsaturated fatty acids, which are known precursors of oxylipins $[16,36]$. WS mainly resulted in increased levels of antioxidant compounds in WTWS and RolCWS plants, as an effect of the induction of ROS molecule production. GRWS plants showed an almost unchanged metabolic profile, confirming the higher resistance of GR-modified plants against abiotic stresses. The results obtained in this study could be useful for further investigations, including the targeted analysis of the most relevant metabolites and additional experiments conducted in field.

Acknowledgments This work was supported by the PRIN grant number 20098TN4CY from the Italian Ministry of Education, University and Research (MIUR). The authors thank Dr. Daniela Almansi for improving English language.

\section{References}

1. Bartwal A, Mall R, Lohani P, Guru SK, Arora S (2013) Role of secondary metabolites and brassinosteroids in plant defense against environmental stresses. J Plant Growth Regul 32:216-232. doi:10. 1007/s00344-012-9272-X

2. Larkindale J, Huang B (2004) Changes of lipid composition and saturation level in leaves and roots for heat-stressed and heatacclimated creeping bentgrass (Agrostis stolonifera). Environ Exp Bot 51:57-67. doi:10.1016/S0098-8472(03)00060-1

3. Yordanov I, Velikova V, Tsonev T (2000) Plant responses to drought, acclimation and stress tolerance. Photosynthetica 38: 171-186. doi:10.1023/A:1007201411474

4. Obata T, Fernie AR (2012) The use of metabolomics to dissect plant responses to abiotic stresses. Cell Mol Life Sci 69:3225-3243. doi: 10.1007/s00018-012-1091-5

5. Ganesh KS, Baskaran L, Rajasekaran S, Sumathi K, Chidambaram AL, Sundaramoorthy P (2008) Chromium stress induced alterations in biochemical and enzyme metabolism in aquatic and terrestrial plants. Colloids Surf B Biointerfaces 63:159-163. doi:10.1016/j. colsurfb.2007.11.016

6. Lipiec J, Doussan C, Nosalewicz A, Kondracka K (2013) Effect of drought and heat stresses on plant growth and yield: a review. Int Agrophysics 27:463-477. doi:10.2478/intag-2013-0017

7. Giannarelli S, Muscatello B, Bogani P, Spiriti MM, Buiatti M, Fuoco R (2010) Comparative determination of some phytohormones in wild-type and genetically modified plants by gas chromatography-mass spectrometry and high-performance liquid chromatography-tandem mass spectrometry. Anal Biochem 398: 60-68. doi:10.1016/j.ab.2009.10.038

8. Irdani T, Caroppo S, Ambrogioni L (2003) Response of Nicotiana tabacum plants overexpressing a glucocorticoid receptor to Meloidogyne incognita (Nematoda tylenchida) infestation. Redia 86:35-38

9. Bettini P, Michelotti S, Bindi D, Giannini R, Capuana M, Buiatti M (2003) Pleiotropic effect of the insertion of the Agrobacterium rhizogenes rolD gene in tomato (Lycopersicon esculentum Mill.). Theor Appl Genet 107:831-836. doi:10.1007/s00122-003-1322-0

10. Palazón J, Cusidó RM, Roig C, Piñol MT (1998) Expression of the rol $\mathrm{C}$ gene and nicotine production in transgenic roots and their regenerated plants. Plant Cell Rep 17:384-390. doi:10.1007/ s002990050411

11. Del Bubba M, Ancillotti C, Checchini L, Ciofi L, Fibbi D, Gonnelli C, Mosti S (2013) Chromium accumulation and changes in plant growth, selected phenolics and sugars of wild type and genetically modified Nicotiana langsdorffii. J Hazard Mater 262:394-403. doi: 10.1016/j.jhazmat.2013.08.073

12. Intrieri MC, Buiatti M (2001) The horizontal transfer of Agrobacterium rhizogenes genes and the evolution of the genus Nicotiana. Mol Phylogenet Evol 20:100-110. doi:10.1006/mpev. 2001.0927

13. Fuoco R, Bogani P, Capodaglio G, Del Bubba M, Abollino O, Giannarelli S, Spiriti MM, Muscatello B, Doumett S, Turetta C, Zangrando R, Zelano V, Buiatti M (2013) Response to metal stress of Nicotiana langsdorffii plants wild-type and transgenic for the rat glucocorticoid receptor gene. J Plant Physiol 170:668-675. doi:10. 1016/j.jplph.2012.12.009

14. Dunn WB, Erban A, Weber RJM, Creek DJ, Brown M, Breitling R, Hankemeier T, Goodacre R, Neumann S, Kopka J, Viant MR (2013) Mass appeal: metabolite identification in mass spectrometry-focused untargeted metabolomics. Metabolomics 9: 44-66. doi:10.1007/s11306-012-0434-4

15. De Vos RCH, Moco S, Lommen A, Keurentjes JJB, Bino RJ, Hall $\mathrm{RD}$ (2007) Untargeted large-scale plant metabolomics using liquid 
chromatography coupled to mass spectrometry. Nat Protoc 2:778791. doi:10.1038/nprot.2007.95

16. Marti G, Erb M, Boccard J, Glauser G, Doyen GR, Villard N, Christelle RAM, Turlings TCJ, Rudaz S, Wolfender J-L (2013) Metabolomics reveals herbivore-induced metabolites of resistance and susceptibility in maize leaves and roots. Plant Cell Environ 36: 621-639. doi:10.1111/pce.12002

17. Lommen A, Kools HJ (2012) MetAlign 3.0: performance enhancement by efficient use of advances in computer hardware. Metabolomics 8:719-726. doi:10.1007/s11306-011-0369-1

18. Lommen A (2009) MetAlign: interface-driven, versatile metabolomics tool for hyphenated full-scan mass spectrometry data preprocessing. Anal Chem 81:3079-3086. doi:10.1021/ac900036d

19. Tikunov YM, Laptenok S, Hall RD, Bovy A, De Vos RCH (2012) MSClust: a tool for unsupervised mass spectra extraction of chromatography-mass spectrometry ion-wise aligned data. Metabolomics 8:714-718. doi:10.1007/s11306-011-0368-2

20. Sumner LW, Amberg A, Barrett D, Beale MH, Beger R, Daykin CA, Fan TW-M, Fiehn O, Goodacre R, Griffin JL, Hankemeier T, Hardy N, Harnly J, Higashi R, Kopka J, Lane AN, Lindon JC, Marriott P, Nicholls AW, Reily MD, Thaden JJ, Viant MR (2007) Proposed minimum reporting standards for chemical analysis. Metabolomics 3:211-221. doi:10.1007/s11306-007-0082-2

21. Sato N, Aoki M, Maru Y, Sonoike K, Minoda A, Tsuzuki M (2003) Involvement of sulfoquinovosyl diacylglycerol in the structural integrity and heat-tolerance of photosystem II. Planta 217:245-251. doi:10.1007/s00425-003-0992-9

22. Chen J, Burke JJ, Xin Z, Xu C, Velten J (2006) Characterization of the Arabidopsis thermosensitive mutant atts 02 reveals an important role for galactolipids in thermotolerance. Plant Cell Environ 29: 1437-1448. doi:10.1111/j.1365-3040.2006.01527.x

23. Burgos A, Szymanski J, Seiwert B, Degenkolbe T, Hannah MA, Giavalisco P, Willmitzer L (2011) Analysis of short-term changes in the Arabidopsis thaliana glycerolipidome in response to temperature and light. Plant J 66:656-668. doi:10.1111/j.1365-313X.2011.04531.x

24. Otsuru M, Yu Y, Mizoi J, Kawamoto-Fujioka M, Wang J, Fujiki Y, Nishida I (2013) Mitochondrial phosphatidylethanolamine level modulates Cyt $\mathrm{c}$ oxidase activity to maintain respiration capacity in Arabidopsis thaliana rosette leaves. Plant Cell Physiol 54:16121619. doi:10.1093/pcp/pct104

25. Kim J, Kang K, Gonzales-Vigil E, Shi F, Jones AD, Barry CS, Last RL (2012) Striking natural diversity in glandular trichome acylsugar composition is shaped by variation at the acyltransferase 2 Locus in the wild tomato Solanum habrochaites. Plant Physiol 160: 1854-1870. doi:10.1104/pp. 112.204735

26. Ghosh B, Westbrook TC, Jones AD (2013) Comparative structural profiling of trichome specialized metabolites in tomato (Solanum lycopersicum) and $\mathrm{S}$. habrochaites: acylsugar profiles revealed by UHPLC / MS and NMR. Metabolomics 10:496-507. doi:10.1007/ s11306-013-0585-y

27. Schilmiller A, Shi F, Kim J, Charbonneau AL, Holmes D, Jones AD, Last RL (2010) Mass spectrometry screening reveals widespread diversity in trichome specialized metabolites of tomato chromosomal substitution lines. Plant J 62:391-403. doi:10.1111/j. 1365-313X.2010.04154.X

28. Pérez-estrada LB, Cano-santana Z, Oyama K (2000) Variation in leaf trichomes of Wigandia urens: environmental factors and physiological consequences. Tree Physiol 20:629-632. doi:10.1093/ treephys/20.9.629

29. Kuc J (1984) Steroid glykoalkaloids and related compounds as potato quality factors. Am Potato J 61:123-139. doi:10.1007/ BF02854034

30. Udalova ZV, Zinov SV, Vasil IS, Paseshnichenko VA (2004) Correlation between the structure of plant steroids and their effects on phytoparasitic nematodes. Appl Biochem Microbiol 40:109113. doi:10.1023/B:ABIM.0000010362.79928.77

31. Coria NA, Sarquı JI, Penalosa I, Urzua M (1998) Heat-induced damage in potato (Solanum tuberosum) tubers: membrane stability, tissue viability, and accumulation of glycoalkaloids. J Agric Food Chem 46:4524-4528. doi:10.1021/jf980151+

32. Dimenstein L, Lisker N, Kedar N, Levy D (1997) Changes in the content of steroidal glycoalkaloids in potato tubers grown in the field and in the greenhouse under different conditions of light, temperature and daylength. Physiol Mol Plant Pathol 50:391-402. doi: 10.1006/pmpp.1997.0098

33. Nitithamyong A, Vonelbe JH, Wheeler RM, Tibbitts TW (1999) Glycoalkaloids in potato tubers grown under controlled environments. Am J Potato Res 76:337-343. doi:10.1007/BF02910006

34. Torras-Claveria L, Jáuregui O, Codina C, Tiburcio AF, Bastida J, Viladomat F (2012) Analysis of phenolic compounds by highperformance liquid chromatography coupled to electrospray ionization tandem mass spectrometry in senescent and water-stressed tobacco. Plant Sci 182:71-78. doi:10.1016/j.plantsci.2011.02.009

35. Van de Mortel J, Schat H, Moerland PD, Van Themaat VEL, Van der Ent S, Blankestijn H, Ghandilyan A, Tsiatsiani S, Aarts MGM (2008) Expression differences for genes involved in lignin, glutathione and sulphate metabolism in response to cadmium in Arabidopsis thaliana and the related $\mathrm{Zn} / \mathrm{Cd}$-hyperaccumulator Thlaspi caerulescens. Plant Cell Environ 31:301-324. doi:10. 1111/j.1365-3040.2007.01764.x

36. Mithöfer A, Schulze B, Boland W (2004) Biotic and heavy metal stress response in plants: evidence for common signals. FEBS Lett 566:1-5. doi:10.1016/j.febslet.2004.04.011

37. Ryu SB, Wang X (1998) Increase in free linolenic and linoleic acids associated with phospholipase D-mediated hydrolysis of phospholipids in wounded castor bean leaves. Biochim Biophys Acta Lipids Lipid Metab 1393:193-202. doi:10.1016/S0005-2760(98)00048-4

38. Bulgakov VP, Aminin DL, Shkryl YN, Gorpenchenko TY, Veremeichik GN, Dmitrenok PS, Zhuravlev YN (2008) Suppression of reactive oxygen species and enhanced stress tolerance in Rubia cordifolia cells expressing the rolC oncogene. Mol Plant Microbe Interact 21:1561-1570. doi:10. 1094/MPMI-21-12-1561 